\title{
Intrinsic Motivation and Real Individual Piecewise Linear Wages
}

\author{
Marie-Christine Thaize Challier \\ Department of Economics, Ermes, University of Paris 2, Paris, France \\ E-mail: marie-christine.thaize@u-paris2.fr \\ Received March 3, 2011; revised April 25, 2011; accepted May 6, 2011
}

\begin{abstract}
This paper presents a simple framework for understanding the prevalence of basic piecewise linear wages in the real world. It extends the analysis of individual piecewise linear wages to environments in which the participation constraint is reinforced by intrinsic motivations. It identifies a class of "acceptable" piecewise linear wages verifying both this reinforced participation constraint and the adverse selection constraint. Among them, it restricts the characterization to a class of real-world wages. Through the advantages and drawbacks of certain acceptable piecewise linear wages, it helps explaining why they are common in the real world even if they are not optimal.
\end{abstract}

Keywords: Real Individual Wages; Piecewise Linear Wages; Intrinsic Motivation

\section{Introduction}

It is widely accepted that simple piecewise linear wages are common in the real world (e.g., Reference [1], p. 9 and References [2-5]). ${ }^{1}$ Indeed linearity is a dominant characteristic of real-world wages since the latter are often (i) very basic remunerations, either constant (such as hourly wages or other time wages) or linear in production (piece wages) or (ii) simple remunerations such as piecewise linear wages. ${ }^{2}$ One of the reasons explaining the importance of such simple wages is their low institutional cost [13]. Another explanation, given by the standard agency model to explicate the practical relevance of such mere wages, considers that risk-neutral principals and agents are indifferent across a large broad range of incentive contracts which are sometimes optimal contracts linear in the outcome (for instance [9]).

The purpose of this paper is to provide an alternative rationale regarding the significance of real piecewise linear wages and not to characterize optimal wages. It clarifies the reason why these wages are of high practical relevance although non optimal. To do this it examines

\footnotetext{
${ }^{1}$ Among the huge literature on the real forms of employees' wages, see also for instance Reference [6] and the special edition on performance and reward [7].

${ }^{2}$ Agency theory has shown that optimal contracts can be linear in the outcome as well as complex nonlinear contracts (e.g., quadratic wages, polynomial schedules). Among the vast literature, see e.g. References [8-10]; and for surveys regarding incentive-based theories see [11] and [12]
}

the properties and implications of some of these realworld piecewise linear wages. It underlines the interest of positive psychology, mainly of intrinsic motivations (see [14-17]) in the labor relations. ${ }^{3}$ Four intrinsic motivations emerges here: self-confidence [23], self-esteem [24], reciprocity [25], and self-efficacy ([26-28]). Another distinguishing mark of our model is due the fact that the threshold is not a predetermined quota as usually set in the literature (References [3-5]). Here, the threshold is endogenous. The present paper is close to the expectancy theory (where employees expect that their efforts will reach the desired performance) discussing the problems caused by poorly defined targets (References [29-32]).

This paper complements the existing theoretical literature on the wage structure. Without any moral hazard constraint, it defines "acceptable" piecewise linear wages, i.e., wages verifying both an adverse selection constraint and an individual rationality constraint reinforced by intrinsic motivations. It limits the study to a class of acceptable wages widespread in the real world and characterizes them. It shows that these non optimal wages can be incentive and highlights their advantages and drawbacks.

Section 2 presents the basic model and defines the family of "acceptable" piecewise linear wages. Section 3 characterizes some real-world wages among this family

\footnotetext{
${ }^{3}$ For economic studies taking into account such personal motivations
} see e.g. References [18-22], and references mentioned below. 
and discusses the implications. Section 4 concludes with a summary.

\section{The Model}

An employer (principal) has to pay a subordinate (agent) possessing private information in a situation characterized by: a static contract in the context of a single period principal-agent framework; a contract whose remuneration depends on an objective measurement of the outcome; and a contract based on the individual performance in the case of a tangible task. Before working, the agent had to choose a contract, knowing not only the technology of the firm and it potential job but also the nature of the output-based pay. The principal and the agent are two risk-neutral parties who reciprocate through a two-part wage system corresponding to piecewise linear wages.

\subsection{Intrinsic Motivation, Participation and Wages}

The agent privately observes his/her ability $\theta \in \Theta$ $=[\underline{\theta}, \bar{\theta}] \subset \mathfrak{R}_{+}$with $\underline{\theta}<\bar{\theta}$. The profit-maximizing principal has some probability distribution for the unknown type parameter $\theta$. The density for this probability, $f(\theta)$, is a continuous function of $\theta$ with $f(\theta)>0$ for all $\theta \in \Theta$. Let $F(\theta)$ be the cumulative distribution function for the parameter $\theta$. The agent exerts a productive effort $e \in A=[0, Z] \subset \mathfrak{R}_{+}$, with $Z>0$, supposed to be an unobservable variable, but its realized outcome is observable for the principal. The cost of effort for the agent having the ability $\theta$ is $c(e, \theta)>0$, twice continuously differentiable in $e$ and $\theta$; it satisfies the usual hypotheses that an increased effort rises the cost whereas an increased ability reduces the cost:

$$
c_{e}>0 ; c_{\theta}<0
$$

The output determines a monetary outcome denoted $y$ with $y \in Y=\left[0, y_{M}\right] \subset \mathfrak{R}_{+}$. It is a random variable with $g(y \mid e, \theta)$ being the probability density function and $G(y \mid e, \theta)$ the cumulative distribution function with the usual hypothesis that, $\forall y$ :

$$
G_{e}(y \mid e, \theta)<0
$$

This means that an additional effort increases the likelihood of higher output. The technology is assumed to be common knowledge.

The principal announces a payment schedule $w$ : $\Theta \times Y \rightarrow \mathfrak{R}_{+}$. When the agent reveals the true type parameter $\theta$ and if the principal observes the production level $y$, the latter pays the amount $w(\theta, y)$ to the agent. Thus the agent's problem is:

$$
\max _{e, \tilde{\theta}} \int_{Y}(w(\tilde{\theta}, y) g(y \mid e, \theta)) \mathrm{d} y-c(e, \theta)
$$

Let $(\hat{e}(\theta, w), \hat{\theta}(\theta, w))$ be the solution of this problem. ${ }^{4}$ Then, the principal solves:

$$
\begin{aligned}
& \max _{w(.)} \int_{\Theta} \int_{Y}(y-w(\tilde{\theta}(\theta, w), y) g(y \mid \hat{e}(\theta, w), \theta)) \\
& \cdot f(\theta) \mathrm{d} y \mathrm{~d} \theta
\end{aligned}
$$

According to Reference [33], the principal can achieve the same expected profit if he chooses $w(\theta,$.$) in order$ to induce the agent to reveal his/her true ability $\theta$. The solution to (3) involves the adverse selection (or truthtelling) incentive constraint:

$$
\hat{\theta}(\theta, w)=\theta, \forall \theta \in \Theta
$$

The principal's problem can thus be stated as:

$$
\max _{w(.)} \int_{\Theta} \int_{Y}(y-w(\theta, y)) g(y \mid \hat{e}(\theta, w), \theta) f(\theta) \mathrm{d} y \mathrm{~d} \theta
$$

under (4). The ex ante participation or individual rationality constraint is given by:

$$
\begin{aligned}
& \int_{Y} w(\tilde{\theta}, y) g(y \mid \hat{e}(\theta, w), \theta) \mathrm{d} y \geq c(\hat{e}(\theta, w), \theta), \\
& \forall \theta \in \Theta
\end{aligned}
$$

Let $t^{*}(\theta)$ be the pure adverse selection transfer defined by Reference [34] as:

$$
t^{*}(\theta)=c(\hat{e}(\theta), \theta)-\int_{\underline{\theta}}^{\theta} c_{s}(\hat{e}(\theta), s) \mathrm{d} s
$$

where the second term of the right-hand side represents the informational rent. This rent is negative by assumption implying that $t^{*}: \Theta \rightarrow \mathfrak{R}_{+}$is a function such that:

$$
t^{*}(\theta) \geq c(\hat{e}(\theta, w), \theta), \forall \theta \in \Theta
$$

The transfer $t^{*}(\theta)$ represents the expected remuneration amount that has to be offered to induce the agent to reveal his/her true type in the presence of adverse selection. The agent faces incentives but still earns $t^{*}(\theta)$ under the optimal effort. Thus, the condition (5) can be reinforced by the following individual rationality constraint: ${ }^{5}$

\footnotetext{
${ }^{4}$ Both the optimal effort $\hat{e}$ and the optimal announcement $\hat{\theta}$ depend on the true ability $\theta$ and on the wage $w$.

${ }^{5}$ Concerning the justification of the wage level and the employer's agreement, it is increasingly recognized that it may be possible to induce the agent to work better by introducing social preferences and reciprocity (References [35,36]). It is also well documented that certain employers are unwilling to accept low wage offers of employees even when unemployment is high (References [37-39]), which is in line with studies in psychology [40]. The sociological version of the efficiency wage theory introduced the idea that the principal may be "generous" in exchange for higher productivity (e.g., References [41,42]). The "fairwage-effort hypothesis" formulates that workers have their own idea about the fair wage and reduce their effort if their actual wage is lower [43].
} 


$$
\int_{Y} w(\tilde{\theta}, y) g(y \mid \hat{e}(\theta, w), \theta) \mathrm{d} y \geq t^{*}(\theta), \forall \theta \in \Theta
$$

A distinguishing mark of this paper appears also through (8), i.e., the individual participation constraint reinforced by non-pecuniary motives that increase the utility reservation. ${ }^{6}$ This constraint encompasses four intrinsic motivations: 1) self-confidence - one's belief in one's abilities; 2) self-esteem - belief regarding a person's evaluation of his/her own worth; 3 ) reciprocal motivation as a "cooperative job attitude" that can affect the compensation package ([25] p. 168); and 4) self-efficacy - belief about future performance; i.e., one's belief in one's capability to achieve certain goals or to one's perception of one's worth to perform well in specific circumstances.

Specifically, first, the reinforced individual participation constraint assumes that the agent not only knows his or her own level of ability but also trusts this level as well as trusts his or her assessment on this level. This refers to self-confidence (see for instance modern psychological theories [23]). The idea here is that the agent self-evaluates, i.e., appraises him-or herself independently and has assurance on his or her own ability's evaluation.

Second, the agent asks for a wage $t *(\theta)$ depending on this ability and assesses this wage as greater than his or her cost $c(\hat{e}(\theta, w), \theta)$. In other words, the agent participates if he or she receives the adverse selection transfer $t *(\theta)$, itself higher than his or her cost. At this stage, our reinforced individual participation constraint refers both to self-esteem and reciprocity. Regarding self-esteem, it underlines that the agent knows not only his or her ability's level but also the value of this ability. He or she is aware of his or her worth. Indeed, according to psychologists (see [24]), self-esteem refers to one's evaluation of his or her own worth. It is a basic human need corresponding to the requirement of self worth from embracing challenges. Concerning reciprocity, the literature (see [25] and the references therein) has shown that reciprocal motivation of employers and employees induce higher effort level and compensation package.

Third, given the level and value of his or her ability, the agent is aware of his or her capacity of reaching the threshold. This idea comes from the theory of self-efficacy reflecting a person's evaluation of his capability of performing well (i.e., attaining a goal, completing tasks, or challenging) [26].

\footnotetext{
${ }^{6}$ In contrast, up to now, the standard model extension about the participation constraint considered that nonmonetary factors lowered the utility reservation. These factors were seen from the point of view of the agent, and included idealism, ethical features, professionalism or mission (References [44,45]). Concerning type-dependent participation constraints in adverse selection models see [46]. On the importance of participation constraints see also [47].
}

\subsection{A Family of Payment Functions}

Consider now a family of piecewise linear wages depending on a vector of parameters $\eta=\left(\alpha_{1}, \alpha_{2}, \beta_{1}, \beta_{2}\right)$ where each parameter is a numerical function on $\Theta$, i.e. $\eta: \Theta \rightarrow \Re_{+}^{4}$. The members of the wage family are defined by the following "two-part wage" system (separating contract) confined to the wages composed of a base salary and a marginal remuneration or bonus per unit of $y$ produced:

$$
\begin{aligned}
& w(\theta, y ; \eta)= \\
& \left\{\begin{array}{c}
w_{1}(\theta, y ; \eta)=\alpha_{1}(\theta) y+\beta_{1}(\theta) \quad \text { if } \quad y<\hat{y}(\theta, \eta) \\
w_{2}(\theta, y ; \eta)=\alpha_{2}(\theta) y+\beta_{2}(\theta) \text { if } \quad y \geq \hat{y}(\theta, \eta)
\end{array}\right.
\end{aligned}
$$

where the threshold $\hat{y}(\theta, \eta) \in Y$ is defined by:

$$
\begin{aligned}
& \int_{0}^{\hat{y}(\theta)}\left(\alpha_{1}(\theta) y+\beta_{1}(\theta)\right) g(y \mid \hat{e}(\theta, \eta), \theta) \mathrm{d} y \\
& +\int_{\hat{y}(\theta)}^{y_{M}}\left(\alpha_{2}(\theta) y+\beta_{2}(\theta)\right) g(y \mid \hat{e}(\theta, \eta), \theta) \mathrm{d} y \\
& =t^{*}(\theta)
\end{aligned}
$$

A relevant point is that (10) stems from the equality between the agent's welfare $E_{y} w()-.c(e, \theta)$ in the real situation and his/her welfare $t^{*}(\theta)-c(e, \theta)$ in the pure adverse selection situation. The constraint (10) comes from (8) when the transfer binds the constraint. Here, the threshold $\hat{y}(\theta, \eta)$ is not a prefixed quota as usually set in the models (References $[3-5,48]$ ). The threshold is here endogenous and results from the assessment of the expected outcome at the point where $E_{y} w()=.t^{*}(\theta)$. Indeed, our principal relies on her costly reciprocity as an incentive device. Put differently, the two part wage system (9) does not refer to the classical incentive device with a prefixed quota. Our consideration is close to the one formulating that firms voluntarily paid rents to elicit non-minimum effort levels (among others, see Reference [49]). In order to elicit more effort, the principal pays more, on the average, if the endogenous threshold is reached (due to the two-part wage constraint) and she pays more than the agent's cost (due to the welfare constraint). Since reciprocity-based voluntary cooperation is an important factor in the labour relationship (for instance, References $[25,35,50])$ I emphasize on the principal's intention to match the expected wage and the pure adverse selection transfer asked by the agent through her individual participation constraint reinforced by her self-efficacy.

Moreover, let us set $w_{2}(\theta, y ; \eta)>w_{1}(\theta, y ; \eta)$ for all $(\theta, y)$, and assume that:

$$
0 \leq \alpha_{1}(\theta)<1 ; 0 \leq \alpha_{2}(\theta)<1 ;
$$




$$
0 \leq \beta_{1}(\theta) \leq \beta_{2}(\theta)
$$

The assumption (11) indicates that the agent cannot be the full residual claimant. This implies (12), that is, entry fees are excluded. In addition, the existence of $\hat{y}(\theta, \eta)$ satisfying (10) depends on the value of $\eta(\theta)$. The family of payment functions should be therefore restricted to $\eta(\theta)$ such that $\hat{y}(\theta, \eta) \in Y$. The value of $\hat{y}(\theta, \eta)$ also depends on $\hat{e}(\theta, \eta)$, the latter being linked to $\eta$ through $w$. Through a minor abuse of notation, $\hat{e}(\theta, w)$ can now be written as $\hat{e}(\theta, \eta)$.

Let $\Omega$ be the family of wages defined by (9), (10), (11) and (12) and such that $\hat{y}(\theta, \eta) \in Y$. By construction, all members of this family satisfy the reinforced participation constraint (8). The definition also assumes that they are incentive compatible, i.e., they induce (4). ${ }^{7}$

The following notation will prove useful. Given $\theta \in \Theta, e \in A$ and a number $y^{*} \in Y$, let $\bar{y}\left(e, \theta, y^{*}\right)$ $=y^{+}\left(e, \theta, y^{*}\right)+y^{-}\left(e, \theta, y^{*}\right)$ be defined by:

$$
\begin{aligned}
& y^{-}\left(e, \theta, y^{*}\right)=\int_{0}^{y^{*}} y g(y \mid e, \theta) \mathrm{d} y \\
& y^{+}\left(e, \theta, y^{*}\right)=\int_{y^{*}}^{y_{M}} y g(y \mid e, \theta) \mathrm{d} y ;
\end{aligned}
$$

Therefore (10) can be written as:

$$
\begin{aligned}
& \alpha_{1}(\theta) y^{-}(.)+\beta_{1}(\theta) G\left(y^{-}(.) \mid \hat{e}(\theta, \eta), \theta\right) \\
& +\alpha_{2}(\theta) y^{+}(.)+\beta_{2}(\theta)\left(1-G\left(y^{-}(.) \mid \hat{e}(\theta, \eta), \theta\right)\right) \\
& =t^{*}(\theta)
\end{aligned}
$$

where, in order to simplify the notation, the parenthesis (.) is explicitly:

$$
(.) \equiv(\hat{e}(\theta, \eta), \theta, \hat{y}(\theta, \eta))
$$

\subsection{On the Agent's Problem}

The agent's problem (3) can now be written as:

$$
\begin{aligned}
& \max _{e \in A} \alpha_{1}(\theta) y^{-}(e, \theta, \hat{y}(\theta, \eta)) \\
& +\beta_{1}(\theta) G\left(y^{-}(e, \theta, \hat{y}(\theta, \eta)) \mid e, \theta\right) \\
& +\alpha_{2}(\theta) y^{+}(e, \theta, \hat{y}(\theta, \eta)) \\
& +\beta_{2}(\theta)\left(1-G\left(y^{-}(e, \theta, \hat{y}(\theta, \eta)) \mid e, \theta\right)\right) \\
& -c(e, \theta)
\end{aligned}
$$

The first order condition for $\hat{e}(\theta, \eta)$ to be a solution of this problem is:

${ }^{7}$ See References [51,52] pp. 257-262.

$$
\begin{aligned}
& \alpha_{1}(\theta) y_{e}^{-}(.)+\beta_{1}(\theta) G_{e}\left(y^{-}(.) \mid \hat{e}(\theta, \eta), \theta\right) \\
& +\alpha_{2}(\theta) y_{e}^{+}(.)-\beta_{2}(\theta)\left(G_{e}\left(y^{-}(.) \mid \hat{e}(\theta, \eta), \theta\right)\right) \\
& =c_{e}(\hat{e}(\theta, \eta), \theta)
\end{aligned}
$$

where (.) is given by (14') and where

$G_{e}\left(y^{-}() \mid. \hat{e}(\theta, \eta), \theta\right)$ is the derivative of the function $G\left(y^{-}() \mid. ., \theta\right)$ evaluated at $\hat{e}(\theta, \eta)$. According to (14), the agent's problem is actually: $\max _{e \in A} t^{*}(\theta)-c(e, \theta)$.

\subsection{A Few Members of $\Omega$}

Let us consider Equations (14) and (16), i.e., the system of two linear equations in the four variables, $\alpha_{1}(\theta)$, $\alpha_{2}(\theta), \beta_{1}(\theta)$ and $\beta_{2}(\theta)$. The set of solutions to this system is a linear variety of dimension 2 in $\mathfrak{R}_{+}^{4}$. There are potentially an infinite number of acceptable members in $\Omega$. The term acceptable means that the piecewise linear wage satisfies (4) and (8). I list some members or subsets of $\Omega$ below; for expositional simplicity, I limit the study to five of them among the above-mentioned real piecewise linear wages. All of them solve (14) and (16). They define an acceptable contract payment function only if they meet the restrictions (11) and (12) and if $\hat{y}(\theta, \eta) \in Y$.

Case 1. $\alpha_{1}(\theta)=\alpha_{2}(\theta)=0$;

$$
\begin{aligned}
& \beta_{1}(\theta)=t^{*}(\theta)+c_{e}(\hat{e}(\theta, \eta), \theta) \\
& \times \frac{1-G\left(y^{-}(e, \theta) \mid e, \theta\right)}{G_{e}\left(y^{-}(\hat{e}(\theta, \eta), \theta, \hat{y}(\theta, \eta)) \mid \hat{e}(\theta, \eta), \theta\right)} ; \\
& \beta_{2}(\theta)=t^{*}(\theta)-c_{e}(\hat{e}(\theta, \eta), \theta) \\
& \times \frac{G\left(y^{-}(e, \theta) \mid e, \theta\right)}{G_{e}\left(y^{-}(\hat{e}(\theta, \eta), \theta, \hat{y}(\theta, \eta)) \mid \hat{e}(\theta, \eta), \theta\right)} .
\end{aligned}
$$

Case 2. $\alpha_{1}(\theta)=0 ; \alpha_{2}(\theta)=\frac{c_{e}(\hat{e}(\theta, \eta), \theta)}{y_{e}^{+}(\hat{e}(\theta, \eta), \theta, \hat{y}(\theta, \eta))}$;

$$
\begin{aligned}
\beta_{1}(\theta)= & \beta_{2}(\theta)=t^{*}(\theta) \\
& -\frac{c_{e}(\hat{e}(\theta, \eta), \theta)}{y_{e}^{+}(\hat{e}(\theta, \eta), \theta, \hat{y}(\theta, \eta))} \\
& y^{+}(\hat{e}(\theta, \eta), \theta, \hat{y}(\theta, \eta)) ;
\end{aligned}
$$

Case 3. The set of solutions to (14) and (16) with $\alpha_{1}(\theta)=0$. For example, if the system is solved for $\alpha_{2}(\theta)$ and $\beta_{2}(\theta)$, we obtain: 


$$
\begin{aligned}
\alpha_{2}(\theta)= & \frac{1}{\Delta}\left\{G_{e}\left(y^{-}(\hat{e}(\theta, \eta), \theta, \hat{y}(\theta, \eta)) \mid \hat{e}(\theta, \eta), \theta\right) \times\left(\beta_{1}(\theta)-t^{*}(\theta)\right)-\left(1-G\left(y^{-}(e, \theta) \mid e, \theta\right)\right) c_{e}(\hat{e}(\theta, \eta), \theta)\right\} \\
& \beta_{2}(\theta)=\frac{1}{\Delta}\left\{\left[\beta_{1}(\theta) G\left(y^{-}(e, \theta) \mid e, \theta\right)-t^{*}(\theta)\right] y_{e}^{+}(\hat{e}(\theta, \eta), \theta, \hat{y}(\theta, \eta))\right. \\
& \left.-\left(\beta_{1}(\theta) G_{e}\left(y^{-}(\hat{e}(\theta, \eta), \theta, \hat{y}(\theta, \eta)) \mid \hat{e}(\theta, \eta), \theta\right)-c_{e}(\hat{e}(\theta, \eta), \theta)\right) y^{+}(\hat{e}(\theta, \eta), \theta, \hat{y}(\theta, \eta))\right\}
\end{aligned}
$$

where $\Delta=-y^{+}(.) G_{e}()-.y_{e}^{+}().(1-G)$.

Case 4. The set of solutions to (14) and (16) with

$\beta_{1}(\theta)=\beta_{2}(\theta)$. The system can be solved for $\alpha_{2}(\theta)$, $\beta_{1}(\theta)$ and $\beta_{2}(\theta)$. This yields:

$$
\begin{gathered}
\alpha_{2}(\theta)=\frac{c_{e}(\hat{e}(\theta, \eta), \theta)-\alpha_{1}(\theta) y_{e}^{-}(e, \theta)}{y_{e}^{+}(\hat{e}(\theta, \eta), \theta, \hat{y}(\theta, \eta))} ; \\
\beta_{1}(\theta)=\beta_{2}(\theta)=t^{*}(\theta)-\alpha_{1}(\theta) y^{-}(e, \theta)-\frac{c_{e}(\hat{e}(\theta, \eta), \theta)-\alpha_{1}(\theta) y_{e}^{-}(e, \theta)}{y_{e}^{+}(\hat{e}(\theta, \eta), \theta, \hat{y}(\theta, \eta))} y^{+}(\hat{e}(\theta, \eta), \theta, \hat{y}(\theta, \eta)) .
\end{gathered}
$$

Setting $\alpha_{1}(\theta)=0$, we get Case 2 .

Case 5. $\alpha_{2}(\theta)=0 ; \beta_{1}(\theta)=0$;

$$
\begin{aligned}
& \alpha_{1}(\theta)=\frac{G_{e}\left(y^{-}(\hat{e}(\theta, \eta), \theta, \hat{y}(\theta, \eta)) \mid \hat{e}(\theta, \eta), \theta\right) t^{*}(\theta)+\left[1-G\left(y^{-}(e, \theta) \mid e, \theta\right] c_{e}(.)\right.}{\left[1-G\left(y^{-}(e, \theta) \mid e, \theta\right)\right] y_{e}^{-}(e, \theta)+G_{e}\left(y^{-}(\hat{e}(\theta, \eta), \theta, \hat{y}(\theta, \eta)) \mid \hat{e}(\theta, \eta), \theta\right) y^{-}(e, \theta)} ; \\
& \beta_{2}(\theta)=\frac{y_{e}^{-}(e, \theta) t *(\theta)-y^{-}(e, \theta) c_{e}(\hat{e}(\theta, \eta), \theta)}{\left[1-G\left(y^{-}(e, \theta) \mid e, \theta\right)\right] y_{e}^{-}(e, \theta)+G_{e}\left(y^{-}(\hat{e}(\theta, \eta), \theta, \hat{y}(\theta, \eta)) \mid \hat{e}(\theta, \eta), \theta\right) y^{-}(e, \theta)} .
\end{aligned}
$$

\section{Characterization and Discussion}

Prior to establishing the propositions, this section defines the following rewards that will be useful to explain some characteristics of the wages.

\section{Definition 1.}

(i) The reward $R(\theta)$ depends on whether $\hat{y}(\theta)$ is reached or not:

$$
R(\theta)=w_{2}(\theta, \hat{y} ; \eta)-w_{1}(\theta, y \prec \hat{y} ; \eta) .{ }^{8}
$$

(ii) The rewards $R_{1}(\theta)$ and $R_{2}(\theta)$ depend on the difference between $w($.$) and t^{*}(\theta)$ :

$$
\begin{aligned}
& R_{1}(\theta)=w_{1}(\theta, y \prec \hat{y} ; \eta)-t^{*}(\theta) ; \\
& R_{2}(\theta)=w_{2}(\theta, \hat{y} ; \eta)-t^{*}(\theta) .
\end{aligned}
$$

To improve the understanding of the rewards $R_{1}(\theta)$ and $R_{2}(\theta)$ the value of $t^{*}(\theta)$ has been positioned in the figures and represented by the horizontal dotted line. ${ }^{9}$

${ }^{8}$ The symbol $\prec$ means here that the value of $y$ is smaller but very close to $\hat{y}$.

${ }^{9}$ The position of $t^{*}(\theta)$ on the figures obviously depends on the steepness of the wages and on the lengths of the segments $[0, \hat{y}(\theta, \eta)[$ and $\left[\hat{y}(\theta, \eta), y_{M}\right]$ where $y_{M}$ is the maximum value of the production $y$.
The following propositions characterize some widespread piecewise linear wages. (All these wages are depicted in the figures displayed in the appendix).

Proposition 1. Let $\eta: \Theta \rightarrow \mathfrak{R}_{+}^{4}, \quad t^{*}(\theta): \Theta \rightarrow \mathfrak{R}_{+}$, and $\hat{y}(\theta, \eta): \Theta \rightarrow \Re$ be functions such that, $\forall \theta \in \Theta$ :

1) The vector $\eta$ has the values given in Case 1 . If these values satisfy (11) and (12), then they define an acceptable wage, called PL1, given by:

$w(\theta, y ; \eta)=$

$$
\left\{\begin{array}{c}
t *(\theta)+\frac{\left(1-G\left(y^{-}(e, \theta) \mid e, \theta\right)\right) c_{e}(\hat{e}(\theta, \eta), \theta)}{G_{e}\left(y^{-}(\hat{e}(\theta, \eta), \theta, \hat{y}(\theta, \eta)) \mid \hat{e}(\theta, \eta), \theta\right)} \\
\quad \text { if } y<\hat{y}(\theta, \eta) \\
t *(\theta)-\frac{G\left(y^{-}(e, \theta) \mid e, \theta\right) c_{e}(\hat{e}(\theta, \eta), \theta)}{G_{e}\left(y^{-}(\hat{e}(\theta, \eta), \theta, \hat{y}(\theta, \eta)) \mid(\hat{e}(\theta, \eta), \theta)\right.} \\
\quad \text { if } y \geq \hat{y}(\theta, \eta)
\end{array}\right.
$$

2) The vector $\eta$ has the values given in Case 2. If these values satisfy (11) and (12), then they define an acceptable wage, called PL2, given by: 


$$
w(\theta, y ; \eta)=\left\{\begin{array}{l}
t^{*}(\theta)-\frac{c_{e}(\hat{e}(\theta, \eta), \theta)}{y_{e}^{+}(\hat{e}(\theta, \eta), \theta, \hat{y}(\theta, \eta))} y^{+}(\hat{e}(e, \theta), \theta, \hat{y}(\theta, \eta)) \quad \text { if } \quad y<\hat{y}(\theta, \eta) \\
t *(\theta)+\frac{c_{e}(\hat{e}(\theta, \eta), \theta)}{y_{e}^{+}(\hat{e}(\theta, \eta), \theta, \hat{y}(\theta, \eta))}\left(y-y^{+}(\hat{e}(\theta, \eta), \theta, \hat{y}(\theta, \eta))\right) \quad \text { if } y \geq \hat{y}(\theta, \eta)
\end{array}\right.
$$

3) The vector $\eta$ has the values given in Case 3. If these values satisfy (11) and (12), then they define an

$$
w(\theta, y ; \eta)=\left\{\begin{array}{lr}
\beta_{1}(\theta) & \text { if } \quad y<\hat{y}(\theta, \eta) \\
\frac{1}{\Delta}\left\{\left[G_{e}(.)\left(\beta_{1}(.)-t^{*}(.)-(1-G(.)) c_{e}(.)\right] y\right.\right. & \\
\left.+\left(\beta_{1}(.) G(.)-t *(.)\right) y_{e}^{+}(.)-\left(\beta_{1}(.) G_{e}(.)-c_{e}(.)\right) y^{+}(.)\right\} & \text {if } \quad y \geq \hat{y}(\theta, \eta)
\end{array}\right.
$$

Proof. In each of these three cases, it is easily verified that the given values for the parameters satisfy (14) and (16).

Implication 1. Proposition 1 and Definition 1 assign the following rewards:

- for wage PL1: $R(\theta)>0, R_{1}(\theta)<0, R_{2}(\theta)>0$.

- for wage PL2: $R(\theta)>0, R_{1}(\theta)<0, R_{2}(\theta)=0$.

- for wage PL3: (a) $R(\theta)>0, R_{1}(\theta)<0, R_{2}(\theta)>0$. (a') $R(\theta)=0, R_{1}(\theta)<0, R_{2}(\theta)<0$.

Proposition 1 refers to acceptable contracts with a constant wage below the threshold $\hat{y}(\theta, \eta)$. The piecewise linear wage PL1 (given in (19) and depicted in Figure 1) includes two constant wages and offers $R(\theta)=$ $-\left(c_{e}(.) / G_{e}().\right)>0 .{ }^{10}$ In addition, $\beta_{2}(\theta)>t *(\theta)$ $>\beta_{1}(\theta)$ under $c_{e}>0$ and $G_{e}<0$. Explicitly, when the real output $y$ is lower (resp. higher) than the threshold $\hat{y}(\theta, \eta)$, the wage received is lower (resp. higher) than the pure adverse selection transfer $t *(\theta)$, the latter being here the reservation wage. It is necessary to produce $y \geq \hat{y}(\theta, \eta)$ in order to obtain $w(\theta, y ; \eta)>t *(\theta)$ (see Table 1). The step-function wage PL1 performs well since it encourages the agent to achieve the threshold. Nevertheless the agent is not stimulated to exceed the threshold since $\beta_{2}(\theta)=0$.

The piecewise linear wages PL2 and PL3 (given in (20) and (21)) present a positive base salary $\beta_{1}(\theta)$, a constant wage below the threshold $\hat{y}(\theta, \eta)$, and a piece wage beyond $\hat{y}(\theta, \eta)$. The wage PL2 (Figure 2) presents a positive reward $R(\theta)$. In addition, if $t^{*}(\theta)$ has the value given in Figure 2 then $R_{1}(\theta)<0$ and $R_{2}(\theta)=0$ (see Implication 1 and Table 1). Put differently, at $y=\hat{y}(\theta, \eta)$, the wage $w_{2}(\theta, y ; \eta)$ will be equal to the pure adverse selection transfer $t *(\theta)$ and thus $R_{2}(\theta)=0$. Therefore, given that the agent asks for

\footnotetext{
${ }^{10}$ The positivity of $R(\theta)$ may be explained by the fact that extra effort both raises the principal's utility via the increase in the probability of reaching the threshold (since $G_{e}<0$ ) and reduces the agent's utility via the increase of the cost ( since $\left.c_{e}>0\right)$.
}

at least $t^{*}(\theta)$, PL2 incite him or her to achieve $\hat{y}(\theta, \eta)$.

The convex wage PL3 may be the noncontinuous convex wage PL3a (see Figure 3a) or the continuous convex wage PL3a' (see Figure 3a'). The analysis is straightforward in the case of the piecewise linear wage PL3a whose nature gives $R(\theta)>0$ and where intrinsic motivations lead the agent to reach the threshold ( $R_{1}(\theta)<0$ and $R_{2}(\theta)>0$ ). In other words, at the threshold $\hat{y}(\theta, \eta)$, the wage received $w(\theta, y ; \eta)$ is higher than the reservation wage $t^{*}(\theta)$ (see Implication 1 and Table 1).

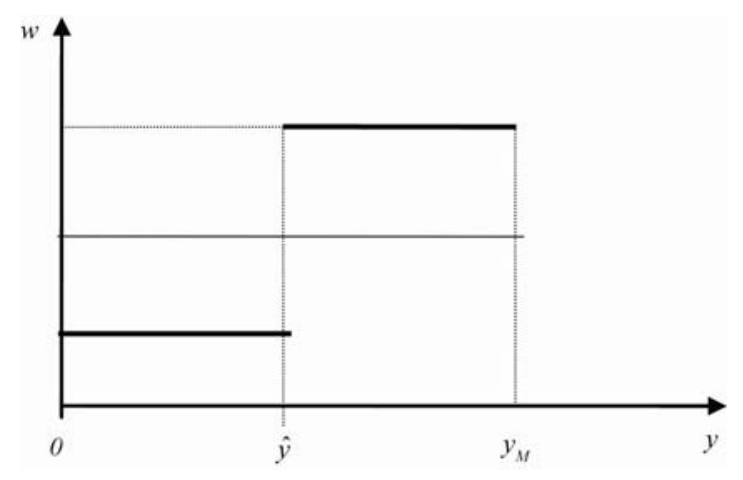

Figure 1. Wage PL1.

Table 1. Summary of the comparison between the real wage and the reservation wage for some of acceptable piecewise linear wages.

\begin{tabular}{ll}
\hline Cases PL1, PL3a, PL4: & $w(\theta, y ; \eta)<t^{*}(\theta)$ at $y<\hat{y}(\theta, \eta) ;$ \\
& $w(\theta, y ; \eta)>t^{*}(\theta)$ at $\hat{y}(\theta, \eta)$ \\
Case PL2: & $w(\theta, y ; \eta)=t^{*}(\theta)$ at $\hat{y}(\theta, \eta)$ \\
Case PL3a': & $w(\theta, y ; \eta)<t^{*}(\theta)$ at $\hat{y}(\theta, \eta)<y<y_{B}$ \\
Case PL5: & $w(\theta, y ; \eta)>t^{*}(\theta)$ at $y_{A}<y<\hat{y}(\theta, \eta)$ \\
\hline
\end{tabular}




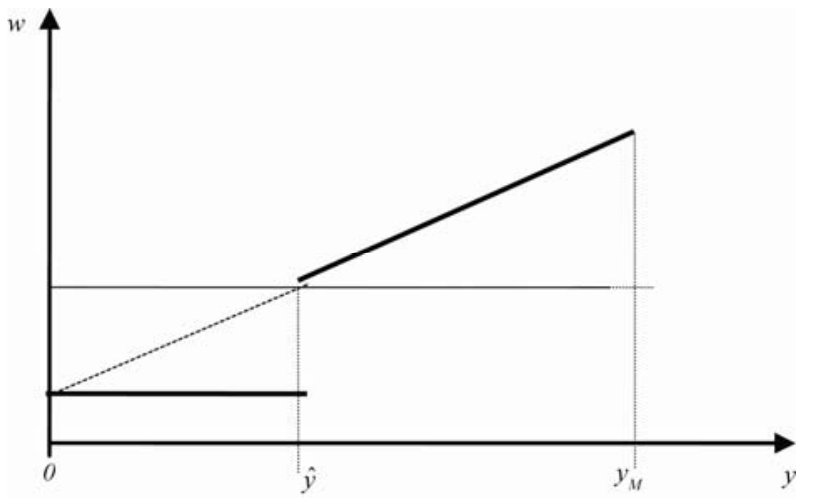

Figure 2. Wage PL2.

In contrast, the wage PL3a' is a continuous wage since there is not a jump of remuneration when $\hat{y}(\theta, \eta)$ is reached; thus $R(\theta)=0$. Second, it corresponds to $w_{2}(\theta, y ; \eta)<t^{*}(\theta)$ even if $\hat{y}(\theta, \eta) \leq y<y_{B}$, implying $R_{2}(\theta)<0 \quad$ (see Implication 1 and Table 1). The wage PL3a' is a very incentive remuneration since the successful agent (who already has reached $\hat{y}(\theta, \eta)$ ) had to ex- ceed $y_{B}$ in order to receive the wage $t *(\theta)$. In other words, PL3a' creates a perverse effect because it penalizes the successful agent who had to exceed the threshold to balance the real wage and the reservation one.

Regarding an application in the case of a US autoglass company, Reference [48] has shown that the transition between the contract PL1 and the one approximating PL3a' (however with $\beta_{2}(\theta)<0$ ) drastically increased the output. Our model explains well this empirical fact. Indeed, although these two contracts elicit a high level of effort, the contract PL1 motivates the agent to reach the threshold $\hat{y}(\theta, \eta)$ in order to receive a wage $w_{2}(\theta, y ; \eta)$ higher than the reservation one $(t *(\theta))$ whereas the contract PL3a' stimulates the agent to reach a higher threshold $y_{B}$ where the real wage $w_{2}(\theta, \hat{y} ; \eta)$ equals $t^{*}(\theta)$.

Proposition 2. Let $\eta: \Theta \rightarrow \mathfrak{R}_{+}^{4}, \quad t^{*}(\theta): \Theta \rightarrow \mathfrak{R}_{+}$, and $\hat{y}(\theta, \eta): \Theta \rightarrow \Re$ be functions such that, $\forall \theta \in \Theta$ :

1) The vector $\eta$ has the values given in Case 4. If these values satisfy (11) and (12), then they define an acceptable wage, called PL4, given by:

$$
w(\theta, y ; \eta)= \begin{cases}t *(\theta)+\alpha_{1}(\theta)\left(y-y^{-}(e, \theta)\right)-\frac{c_{e}(\hat{e}(\theta, \eta), \theta)-\alpha_{1}(\theta) y_{e}^{-}(e, \theta)}{y_{e}^{+}(\hat{e}(\theta, \eta), \theta, \hat{y}(\theta, \eta))} y^{+}(\cdot) & \text { if } y<\hat{y}(\theta, \eta) \\ t *(\theta)-\alpha_{1}(\theta) y^{-}(e, \theta)+\frac{c_{e}(\hat{e}(\theta, \eta), \theta)-\alpha_{1}(\theta) y_{e}^{-}(e, \theta)}{y_{e}^{+}(\hat{e}(\theta, \eta), \theta, \hat{y}(\theta, \eta))}\left(y-y^{+}(\cdot)\right) & \text { if } y \geq \hat{y}(\theta, \eta)\end{cases}
$$

2) The vector $\eta$ has the values given in Case 5. If these values satisfy (11) and (12), then they define an

$$
w(\theta, y ; \eta)= \begin{cases}\frac{G_{e}\left(y^{-}(.) \mid \hat{e}(\theta, \eta), \theta\right) t *(\theta)+\left(1-G\left(y^{-}(.) \mid e, \theta\right)\right) c_{e}(.)}{\left(1-G\left(y^{-}(.) \mid e, \theta\right)\right) y_{e}^{-}(e, \theta)+\left(G_{e}\left(y^{-}(.) \mid \hat{e}(\theta, \eta), \theta\right)\right) y^{-}(e, \theta)} & \text { if } \quad y<\hat{y}(\theta, \eta) \\ \frac{y_{e}^{-}(e, \theta) t^{*}(\theta)-y^{-}(e, \theta) c_{e}(.)}{\left(1-G\left(y^{-}(.) \mid e, \theta\right)\right) y_{e}^{-}(e, \theta)+\left(G_{e}\left(y^{-}(.) \mid \hat{e}(\theta, \eta), \theta\right)\right) y^{-}(e, \theta)} & \text { if } \quad y \geq \hat{y}(\theta, \eta) .\end{cases}
$$

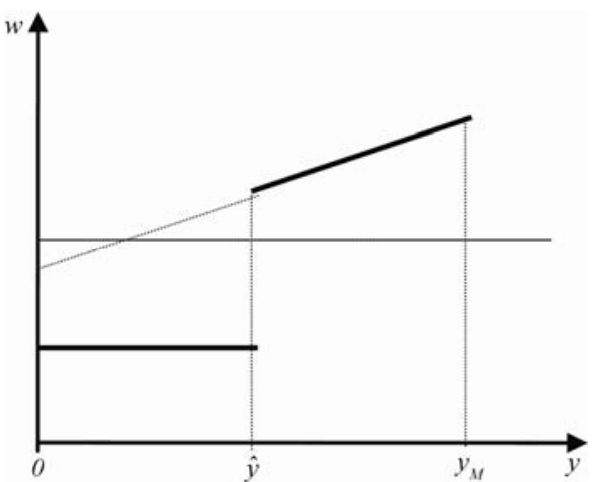

(a)

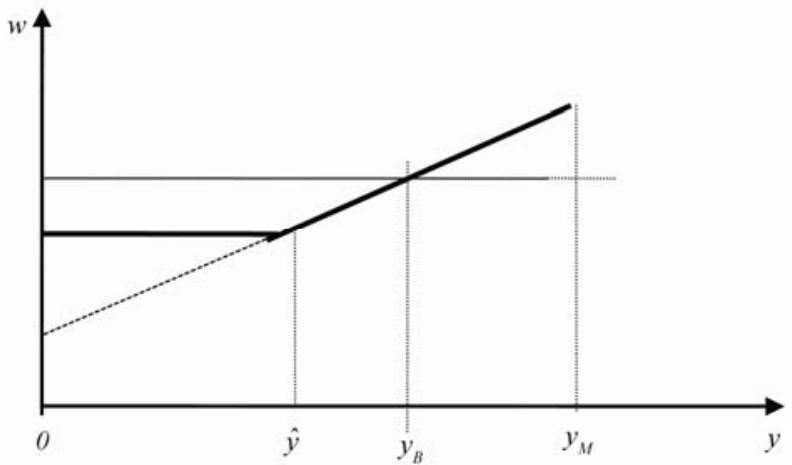

(a')

Figure 3. (a) Wage PL3a; (b) Wage PL3a'. 
Proof. The values for the parameters satisfy (14) and (16). The proof is straightforward.

Implication 2. Proposition 2 and Definition 1 assign the following rewards:

- for wage PL4: $R(\theta)>0, R_{1}(\theta)<0, R_{2}(\theta)>0$.

- for wage PL5: $R(\theta)=0, R_{1}(\theta)>0$ between $y_{A}$ and $\hat{y}(\theta, \eta), R_{2}(\theta)>0$.

Proposition 2 deals with acceptable contracts without a constant wage below the threshold. The piecewise linear wage PL4 (given in (22) and represented in Figure 4) includes a positive base salary $(\beta(\theta)>0)$ and two increasing wages. It is incentive not only because the agent receives the reward $R(\theta)>0$ if $y \geq \hat{y}(\theta, \eta)$ and is incrementally penalized below $\hat{y}(\theta, \eta)$ but also because it encourages the agent to reach $\hat{y}(\theta, \eta)$ in order to obtain more than $t *(\theta)$.

Up to now, the agent who satisfies his or her intrinsic motivations had to attain the threshold (wages PL1, PL2, PL3a, and PL4) or to exceed it (wage PL3a'). Such wages are incentive. This explains why they are common in the concrete world despite their non-optimality. In contrast, the wage PL5 is presented here as a counterexample regarding the value of $R_{1}(\theta)$ (Implication 2). The continuous concave wage PL5 (given in (23) and depicted in Figure 5) is composed of a base salary equals to zero, an increasing wage until the threshold, and a constant compensation beyond it. Even if the threshold is not reached, the agent receives a remuneration higher than the pure adverse selection transfer. In formal terms, if $y_{A} \leq y<\hat{y}(\theta, \eta)$ then $w_{1}(\theta, y ; \eta)>t *(\theta)$. Otherwise stated, although PL5 satisfies the reinforced participation constraint and the adverse selection constraint, the agent is not stimulated to achieve the threshold $\hat{y}(\theta, \eta)$ since his or her reservation wage $t^{*}(\theta)$ is already satisfied at $y_{A}$ (see Implication 2 and Table 1). This is the type of perverse effect where the real wage favors the agent who does not reach the threshold.

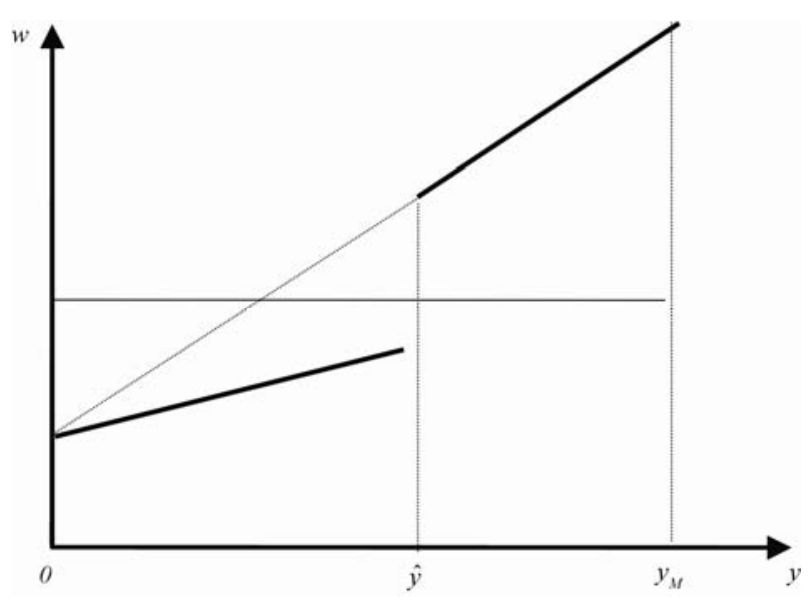

Figure 4. Wage PL4.

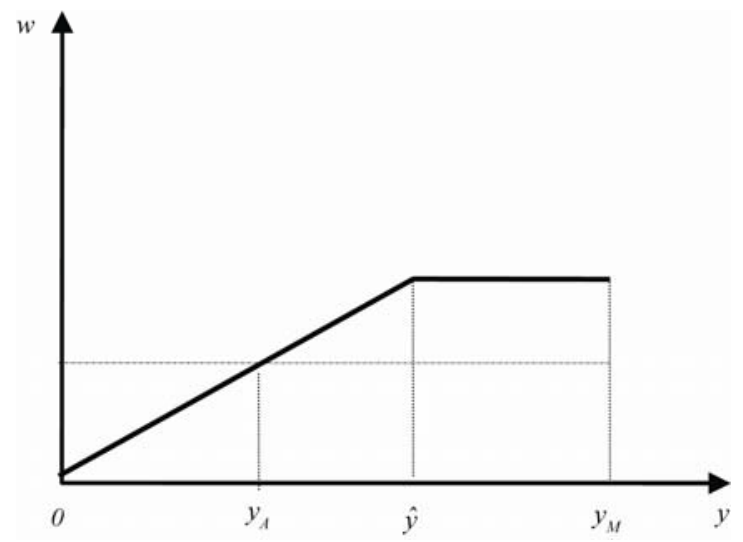

Figure 5. Wage PL5.

To sum up, among the infinite number of acceptable wages, the paper has selected and characterized six wages found in the real world. Five of these six wages are shown to be incentive (PL1, PL2, PL3a, PL3a', and PL4). However, the incentive continuous convex wage PL3a' creates a perverse effect since it penalizes the successful agent who had to exceed the threshold to balance the real wage and the reservation one. Finally, the concave wage PL5 is not incentive; it induces a second perverse effect where the agent who does not reach the threshold is rewarded.

The findings of this paper should not be interpreted to endorse the generalization of output-contingent compensations. They only help explaining the real-world piecewise linear wages. This empirical fact may be understood through the advantages and drawbacks of such payments. Among their advantages, the following ones are noteworthy. Regarding the agent, the individual participation constraint is reinforced by non-pecuniary motivations that increase the reservation wage. A second advantage occurs as regards the wages PL1, PL3a and PL4 since, at $\hat{y}(\theta, \eta)$, the agent enjoys a wage $w(\theta, y ; \eta)$ strictly higher than the reservation wage $t^{*}(\theta)$. (Concerning the wage PL5, $w(\theta, y ; \eta)$ is even strictly higher than $t^{*}(\theta)$ before reaching the threshold). As regards the drawbacks of these wages, the findings have highlighted two perverse effects. A first drawback relates to the principal through the wage PL5 whereas the second one affects the agent through the wage PL3a'.

\section{Conclusions}

Through a simple model, this paper contributes to the understanding of the prevalence of several non optimal piecewise linear wages. It enriches theories of compensation by hypothesizing new determinants of pay (through intrinsic motivations reinforcing the classical individual participation constraint) and by identifying and 
characterizing some subsequent "acceptable" piecewise linear wages. By definition, the term "acceptable" means that the wages verify the adverse selection constraint and a participation constraint reinforced by intrinsic motivations such as agent's self-confidence, self-esteem, reciprocity, and self-efficacy. Then, the paper defines the wage structure - noncontinuity or continuity, convexity or concavity, that is, the compensation gap (i.e., positive or negative rewards) between the real wages and the reservation ones. It shows that some acceptable piecewise linear wages can be incentive.

The recent years saw a significant increase in the use of simple output-contingent payments over the world. Thus, the present theoretical findings may explain this empirical fact through the advantages and drawbacks of such payments.

\section{Acknowledgements}

I am grateful to Michel Truchon for generous and very helpful comments and suggestions. I also benefited from the comments of the conference participants at the European Economic Association meeting at Budapest and of the seminar participants at University of Laval on earlier drafts of this paper. Of course, the usual caveats apply.

\section{References}

[1] O. D. Hart and B. Holmström, "The Theory of Contracts," Advances in Economic Theory: Fifth World Congress, Cambridge University Press, Cambridge, 1987. doi:10.1017/CCOL0521340446.003

[2] E. F. Fama, "Time, Salary, and Incentive Payoffs in Labor Contracts," Journal of Labor Economics, Vol. 9, No 1, 1991, pp. 25-44. doi:10.1086/298257

[3] E. P. Lazear, "Personnel Economics," The MIT Press, Cambridge, 1996.

[4] E. P. Lazear, "Personnel Economics for Managers," Wiley, New York, 1998.

[5] E. P. Lazear, "Personnel Economics: Past Lessons and Future Directions," Journal of Labor Economics, Vol. 17, No. 2, 1999, pp. 199-236. doi:10.1086/209918

[6] D. Parent, "Methods of Pay and Earnings: A Longitudinal Analysis," Industrial and Labor Relations Review, Vol. 53, No. 1, 1999, pp. 71-86. doi:10.2307/2696162

[7] D. Collings, T. Dundon and M. Marchington (Eds.), "Human Resource Management Journal," Special Edition on Performance and Reward, Vol. 18, No. 4, 2008.

[8] P. Picard, "On the Design of Incentive Schemes under Moral Hazard and Adverse Selection," Journal of Public Economics, Vol. 33, No. 3, 1987, pp. 305-331. doi:10.1016/0047-2727(87)90058-2

[9] J.-J. Laffont and J. Tirole, "The Dynamics of Incentive Contracts," Econometrica, Vol. 56, No. 5, 1988, pp. 11531175. doi: $10.2307 / 1911362$
[10] F. Balmaceda, "Uncertainty, Pay for Performance, and Asymmetric Information," Journal of Law, Economics and Organization, Vol. 25, No. 2, 2009, pp. 400-441. doi:10.1093/jleo/ewn022

[11] R. Gibbons, "Incentives in Organizations," Journal of Economic Perspectives, Vol. 12, No. 4, 1998, pp. 115132.

[12] C. Prendergast, "The Provision of Incentives in Firms," Journal of Economic Literature, Vol. 37, 1999, pp. 7-63. doi: $10.1257 /$ jel.37.1.7

[13] B. Holmström and P. Milgrom, "Aggregation and Linearity in the Provision of Intertemporal Incentives," Econometrica, Vol. 55, No. 2, 1987, pp. 303-328. doi: $10.2307 / 1913238$

[14] E. L. Deci, "Intrinsic Motivation," Plenum Press, New York, 1975.

[15] E. L. Deci and R. M. Ryan, "Intrinsic Motivation and Self-Determination in Human Behavior," Plenum Press, New York, 1985.

[16] M. E. P. Seligman, "Authentic Happiness: Using the New Positive Psychology to Realize Your Potential for Lasting Fulfillment," Free Press, New York, 2002.

[17] C. Peterson and M. E. P. Seligman, "Character Strengths and Virtues: A Handbook and Classification," Oxford University Press, Oxford, 2004.

[18] B. Frey, "Not Just for the Money. An Economic Theory of Personal Motivation," Edward Elgar, Cheltenham, 1997.

[19] D. Kreps, "Intrinsic Motivation and Extrinsic Incentives," American Economic Review, Vol. 87, No. 2, 1997, pp. 359-364.

[20] U. Gneezy and A. Rustichini, "Pay Enough or Don't Pay at All," Quarterly Journal of Economics, Vol. 115, No. 3, 2000, pp. 791-810. doi:10.1162/003355300554917

[21] R. Bénabou and J. Tirole, "Intrinsic and Extrinsic Motivation," Review of Economic Studies, Vol. 70, 2003, pp. 489-520.

[22] R. Bénabou and J. Tirole, "Incentives and Prosocial Behavior," American Economic Review, Vol. 96, No. 5, 2006, pp. 1652-1678.

[23] R. J. Corsini, "The Dictionary of Psychology," Routledge, Brunner, 1999.

[24] N. Branden, "The Psychology of Self-Esteem," Bantam, New York, 1969.

[25] E. Fehr and S. Gachter, "Fairness and Retaliation: The Economics of Reciprocity," Journal of Economic Perpectives, Vol. 14, No. 3, 2000, pp. 159-181. doi:10.1257/jep.14.3.159

[26] A. Bandura, "Self-Efficacy: The Exercise of Control," Freeman \& Co., New York, 1997.

[27] A. Bandura and E. A. Locke, "Negative Self-Efficacy and Goal Effects Revisited," Journal of Applied Psychology, Vol. 88, No. 1, 2003, pp. 87-99. doi:10.1037/0021-9010.88.1.87

[28] J. B. Vancouver, K. M. More and R. J. Yoder, "Self-Efficacy and Resource Allocation: Support for a Discontinuous Model," Journal of Applied Psychology, Vol. 93, 
No. 1, 2008, pp. 35-47. doi:10.1037/0021-9010.93.1.35

[29] V. H. Wroom, "Work and Motivation," Wiley, New York, 1964.

[30] L. W. Porter and E. E. Lawler, "Managerial Attitudes and Performance," Dorsey, Homewood, 1968.

[31] E. A. Locke and G. P. Latham, "A Theory of Goal Setting and Task Performance," Prentice-Hall, Englewood Cliffs, 1990.

[32] A. Furnham, "The Psychology of Behavior at Work: The Individual in the Organization," Psychology Press, Hove, 1997.

[33] R. B. Myerson, "Optimal Coordination Mechanisms in Generalized Principal-Agent Problems," Journal of Mathematical Economics, Vol. 10, 1982, pp. 67-81. doi:10.1016/0304-4068(82)90006-4

[34] J. A. Mirrlees, "An Exploration in the Theory of Optimum Income Taxation," Review of Economic Studies, Vol. 38, No. 2, 1971, pp. 175-208. doi:10.2307/2296779

[35] E. Fehr, S. Gächter and G. Kirchsteiger, "Reciprocal Fairness and Noncompensating Wage Differentials," Journal of Institutional and Theoretical Economics, Vol. 152, No. 4, 1996, pp. 608-640.

[36] E. Fehr and K. M. Schmidt, "Fairness, Incentives, and Contractual Choices," European Economic Review, Vol. 44, No. 4-6, 2000, pp. 1057-1068. doi:10.1016/S0014-2921(99)00046-X

[37] T. F. Bewley, "Why Not Cut Pay?" European Economic Review, Vol. 42, No. 2, 1998, pp. 459-490. doi:10.1016/S0014-2921(98)00002-6

[38] E. Fehr and A. Falk, "Wage Rigidity in a Competitive Incomplete Contract Market," Journal of Political Economy, Vol. 107, No. 1, 1999, pp. 106-134. doi:10.1086/250052

[39] M. Dufwenberg and G. Kirchsteiger, "Reciprocity and Wage Undercutting," European Economic Review, Vol. 44, No. 4-6, 2000, pp. 1069-1078. doi:10.1016/S0014-2921(99)00047-1

[40] M. Argyle, "The Social Psychology of Work," 2nd Edtion, Penguin, Harmondsworth, 1989.
[41] G. A. Akerlof, "Labor Contracts as Partial Gift Exchange," Quarterly Journal of Economics, Vol. 97, No. 4, 1982, pp. 543-569. doi:10.2307/1885099

[42] G. A. Akerlof and J. Yellen, "Efficiency Wage Models of the Labor Market," Cambridge University Press, Cambridge, 1986. doi:10.1017/CBO9780511559594

[43] G. A. Akerlof and J. Yellen, "The Fair Wage-Effort Hypothesis and Unemployment," Quarterly Journal of Economics, Vol. 105, No. 2, 1990, pp. 255-283. doi: $10.2307 / 2937787$

[44] J. Q. Wilson, "Bureaucracy: What Government Agencies Do and Why They Do it," Basic Books, New York, 1989.

[45] T. Besley and M. Ghatak, "Competition and Incentives with Motivated Agents," American Economic Review, Vol. 95, No. 3, 2005, pp. 616-636. doi: $10.1257 / 0002828054201413$

[46] B. Jullien, "Participation Constraints in Adverse Selection Models," Journal of Economic Theory, Vol. 93, No. 1, 2000, pp. 1-47. doi:10.1006/jeth.1999.2641

[47] P. Oyer, "Why Do Firms Use Incentives That Have No Incentive Effects," Journal of Finance, Vol. 59, No. 4, 2004, pp. 1619-1650. doi:10.1111/j.1540-6261.2004.00674.x

[48] E. P. Lazear, "Performance Pay and Productivity," American Economic Review, Vol. 90, No. 5, 2000, pp. 13461361. doi:10.1257/aer.90.5.1346

[49] E. Fehr, E. Kirchler, A. Weichbold and S. Gächter, "When Social Norms Overpower Competition: Gift Exchange in Experimental Labor Markets," Journal of Labor Economics, Vol. 16, No. 2, 1998, pp. 324-351. doi:10.1086/209891

[50] E. Fehr and A. Falk, "Psychological Foundations of Incentives," European Economic Review, Vol. 46, No. 4-5, 2002, pp. 687-724. doi:10.1016/S0014-2921(01)00208-2

[51] J.-J. Laffont and J. Tirole, "Using Cost Observation to Regulate Firms," Journal of Political Economy, Vol. 94, No. 3, 1986, pp. 614-641. doi:10.1086/261392

[52] D. Fudenberg and J. Tirole, "Game Theory," MIT Press, Cambridge, 1991. 